TITLE:

\title{
Study of Electron Orbits for Formation of Toroidal Closed Flux Surface by ECH
}

\section{$\operatorname{AUTHOR}(\mathrm{S}):$}

Maekawa, Takashi; Yoshinaga, Tomokazu; Tanaka, Hitoshi; Uchida, Masaki; Watanabe, Fumitake

\section{CITATION:}

Maekawa, Takashi ...[et al]. Study of Electron Orbits for Formation of Toroidal Closed Flux Surface by ECH. Plasma Science and Technology 2011, 13(3): 342-346

\section{ISSUE DATE:}

2011-06-01

URL:

http://hdl.handle.net/2433/143688

\section{RIGHT:}

C IOP Publishing 2011; この論文は出版社版でありません。引用の際に は出版社版をご確認ご利用ください。; This is not the published version. Please cite only the published version. 


\title{
Study of Electron Orbits for Formation of Toroidal Closed Flux Surface by ECH MAEKAWA Takashi ${ }^{1}$, YOSHINAGA Tomokazu ${ }^{2}$, TANAKA Hitoshi ${ }^{1}$, UCHIDA Masaki $^{1}$, WATANABE Fumitake ${ }^{1}$ \\ ${ }^{1}$ Graduate School of Energy Science, Kyoto University, Kyoto 606-8502, Japan \\ ${ }^{2}$ National Institute for Fusion Science, Toki 509-5292, Japan
}

\begin{abstract}
Electron orbits under the external vertical field $\left(\mathrm{B}_{\mathrm{V}}\right)$ and the self poloidal field of the toroidal plasma current are investigated by using analytic model fields in order to search for the conditions of appearance of appropriate confinement asymmetry of fast electrons along the field lines. This asymmetry efficiently and quickly generates a toroidal current, which may close the field lines in ECH-started plasmas. It is analytically shown that the characteristics of confinement asymmetry depend on the product of the major radius and the strength of the external vertical field. The results suggest that in large devices $\mathrm{B}_{\mathrm{V}}$ should be lowered and also some artificial means of quickening the pitch angle scattering for the fast electrons at higher energy range is beneficial to generate the toroidal current for closing the filed lines.
\end{abstract}

Keywords: ECH, closed flux surface, Electron orbit,

PACS: 52.55.Fa, 52.35.Vd, 52.50.Sw

\section{Introduction}

Removal of central solenoid from the core of tokamak reactors is beneficial ${ }^{[1]}$. They would be reduced in size, simplified in structure. Furthermore, plasma performance would be improved via reduction of the aspect ratio, and, therefore, the construction cost of the reactors could be reduced. Without the central solenoid, however, alternative methods for plasma initiation and current start up are required. The electron cyclotron heating $(\mathrm{ECH})$ is potentially an attractive candidate for this purpose in reactors since the microwaves for ECH can be launched with a simple small launcher remotely positioned from the plasma surface.

Recently, a closed flux surface was found to be formed via current jump by ECH under a weak vertical field $B_{v}$ in small and low-aspect-ratio torus devices, the LATE device ${ }^{[2]}$, the CPD device ${ }^{[3]}$ and the SUNIST device ${ }^{[4]}$. Furthermore, the plasma current was ramped up by EC current drive (ECCD) after the formation of closed flux surface in the LATE device ${ }^{[5,6]}$. Then a 
question arises; whether such a closing of flux surface by ECH is also possible or not in large devices. In other words what is needed in the large devices to ensure closing of the field lines.

A model for closing of flux surface via current jump was briefly proposed in [2]. The model predicts that unidirectional confined orbits of fast electrons, which arise prior to the change of the magnetic field from open to closed structure, leads to a quick increase of current, that is, current jump. However, there has been no study how the model depends on the scale of the device. In the present paper we investigate the scale dependence of the model and discuss the implications of the results.

\section{Electron Orbits and Discussions}

We employ cylindrical coordinates as shown in figure 1 . In a simple toroidal field $B_{\phi}$, electrons drift vertically by the curvature and gradient of the field. The vertical drift velocity is given by,

$V_{Z}=\frac{m \gamma\left(v_{\| /}^{2}+v_{\perp}^{2} / 2\right)}{q R B_{\phi}}$.

Here $\gamma$ is the relativistic factor, $q$ and $m$ are electron mass and charge, respectively, and the subscripts // and $\perp$ denote parallel and perpendicular components of the electron velocity to the magnetic field, respectively. The positive ions also drift vertically, but the direction is opposite to the electron drift direction. Thus there arises vertical charge separation. When a weak vertical field $\mathrm{B}_{\mathrm{V}}$ is superposed in addition to the toroidal field, the field lines become helical. Then, the electrons return along the helical field lines to compensate the charge separation, generating a toroidal current. The current density is proportional to the electron pressure as follows,

$j_{\phi}=-\frac{2 P_{e}}{R B_{V}}$.

This was originally proposed as an equilibrium current by the fluid model ${ }^{[7]}$ and recently confirmed experimentally ${ }^{[8]}$.

On the other hand, once closed flux surfaces are formed, a unidirectional tail electrons by ECCD may carry the toroidal current. Thus, the current generation mechanisms are quite different between under the open and closed field structures. Therefore, there must be third mechanism of current generation that bridges these two regimes of different current generations. Confinement asymmetry of electron orbits along the field lines, which is enhanced when the field changes 
significantly from the vacuum field by the pressure driven current (equation (2)), has been invoked as the third mechanism ${ }^{[2]}$.

\section{(Figure 1)}

Let us begin with the simplest case of electron orbits, that is, orbits in a simple toroidal field. In this case the electrons drift vertically as predicted by equation (1) and, therefore, there is no confined orbit. When a weak vertical field $\mathrm{B}_{\mathrm{V}}$ is superposed to the toroidal field $B_{\phi}$, another vertical velocity component arises from the parallel drift velocity along the helical field lines in addition to the vertical charge separation drift. The total vertical drift velocity is given by

$$
V_{Z}=v_{/ /} \frac{B_{V}}{B_{\phi}}-\frac{m \gamma\left(v_{/ /}^{2}+v_{\perp}^{2} / 2\right)}{q R B_{\phi}}
$$

The electrons which satisfy the condition, $\mathrm{V}_{\mathrm{Z}}=0$ in equation (3), have confined orbits. This condition reduces to

$$
\left[1+\left.\left(\frac{R \Omega_{V}}{c}\right)^{2}\right|_{\rfloor}\left(\frac{v_{I I}}{c}\right)^{4}+\left\{\left[1+\left.\left(\frac{R \Omega_{V}}{c}\right)^{2}\right|_{\mid}\left|\left(\frac{v_{\perp}}{c}\right)^{2}-\left(\frac{R \Omega_{V}}{c}\right)^{2}\right|_{\mid}\left(\frac{v_{I I}}{c}\right)^{2}+\frac{1}{4}\left(\frac{v_{\perp}}{c}\right)^{4}=0\right.\right.\right.
$$

, where $\Omega_{V}=e B_{V} / m$. In the non relativistic case, the above equation is simplified as

$$
\frac{\left(v_{/ /}-\frac{R \Omega_{V}}{2}\right)^{2}}{\left(\frac{R \Omega_{V}}{2}\right)^{2}}+\frac{v_{\perp}{ }^{2}}{2\left(\frac{R \Omega_{V}}{2}\right)^{2}}=1
$$

This relationship makes an ellipse in the electron velocity space that is referred as $V_{Z}=0$ ellipse. It is noted that the product $R B_{V}$ uniquely determines the $V_{Z}=0$ characteristics given by equations (4) and (5). Some examples of $\mathrm{V}_{\mathrm{Z}}=0$ ellipses are plotted in figures 2 and 3, manifesting asymmetric confinement along the field lines in the particle orbit picture.

In order to study characteristics of electron orbits under non-uniform $\mathrm{B}_{\mathrm{V}}$ and the self poloidal field due to the toroidal plasma current, we have developed analytic models for the $\mathrm{B}_{\mathrm{V}}$ and the self poloidal field. The flux function of $\mathrm{B}_{\mathrm{V}}$ field may be given by,

$$
\Psi_{e x t}=R_{0} X B_{V 0}+\frac{1-n}{2} X^{2} B_{V 0}+\frac{n}{2} Z^{2} B_{V 0}+\left(\frac{1}{6} \Psi_{X X X} X^{3}+\frac{1}{2} \Psi_{X Z Z} X Z^{2}\right)
$$

, where $X=R-R_{0}, \quad \Psi_{X X X}=\partial^{3} \Psi_{e x t} /\left.\partial X^{3}\right|_{R=R_{0}, Z=0}, \quad \Psi_{X Z Z}=\partial^{3} \Psi_{e x t} /\left.\partial X \partial Z^{2}\right|_{R=R_{0}, Z=0}$, 
$\mathrm{B}_{\mathrm{V} 0}$ is the vertical field at $\mathrm{R}=\mathrm{R}_{0}$, and $\mathrm{n}$ is the decay index of $\mathrm{B}_{\mathrm{V}}$ at $\mathrm{R}=\mathrm{R}_{0}$, $n=-\left(R_{0} / B_{V 0}\right) \partial B_{V} /\left.\partial R\right|_{R=R_{0}}$. Using normalized forms of

$\psi_{\text {ext }}=\Psi_{\text {ext }} / R_{0}^{2} B_{V 0}, x=X / R_{0}$ and $z=Z / R_{0}$, equation (6) reduces to

$\psi_{\text {ext }}=x+\frac{1-n}{2} x^{2}+\frac{n}{2} z^{2}+\left(\frac{1}{6} \psi_{x x x} x^{3}+\frac{1}{2} \psi_{x z z} x z^{2}\right)$

While this form generates finite toroidal current density inside the minor radius, it becomes negligible by setting $\psi_{x x x}=-n$ and $\psi_{x z z}=0$.

The plasma current profile assumes the following form ${ }^{[9]}$

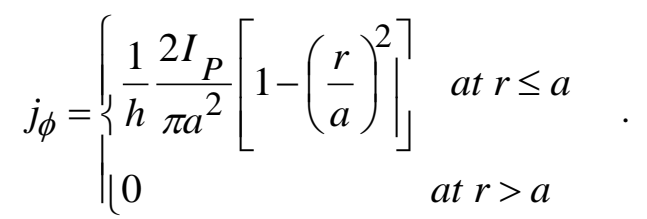

Here, $h=1+r \cos \theta / R_{0}$ and $r=\sqrt{X^{2}+Z^{2}}$. Then the self poloidal field takes the form, $\mathbf{B}=B_{\theta} / h \hat{\theta}$, where

$$
B_{\theta}= \begin{cases}2 B_{a}\left[\frac{r}{a}-\left.\frac{1}{2}\left(\frac{r}{a}\right)^{3}\right|_{\mid}\right. & \text {at } r \leq a \\ B_{a}\left(\frac{r}{a}\right)^{-1} & \text { at } r>a\end{cases}
$$

Here, $B_{a}=-\mu_{0} I_{P} / 2 \pi a$ is the field strength at the current boundary $(r=a)$.

The flux function of this self field in the normalized form is given by

$$
\psi_{\text {int }} \equiv \frac{\Psi_{\text {int }}}{R_{0}^{2} B_{V 0}}= \begin{cases}\frac{a}{R_{0}} \frac{B_{a}}{B_{V 0}}\left[\left(\frac{r / R_{0}}{a / R_{0}}\right)^{2}-\frac{1}{4}\left(\frac{r / R_{0}}{a / R_{0}}\right)^{4}\right\rceil \text { at } r \leq a \\ \frac{a}{R_{0}} \frac{B_{a}}{B_{V 0}}\left[\frac{3}{4}+\log \left(\frac{r / R_{0}}{a / R_{0}}\right)\right\rfloor & \text { at } r>a\end{cases}
$$

There are three constants for the motion of electron in above axisymmetric fields; the angular momentum, energy (square of velocity) and magnetic moment as follows, respectively,

$$
\begin{aligned}
& P_{\phi}=m \gamma R v_{\phi}+q\left(\Psi_{\text {ext }}+\Psi_{\mathrm{int}}\right) \cong m \gamma R v_{/ /}+q\left(\Psi_{\text {ext }}+\Psi_{\mathrm{int}}\right) \\
& W=v_{\perp}^{2}+v_{/ /}^{2} \\
& \mu=\frac{m \gamma v_{\perp}^{2}}{2 B} \cong \frac{m v_{\perp}^{2}}{2 B_{\phi}}
\end{aligned}
$$


Then,

$W=\frac{2}{m \gamma} \mu B_{\phi}+\frac{1}{2}\left[\left.\frac{P_{\phi}-q\left(\Psi_{e x t}+\Psi_{\text {int }}\right)}{m \gamma R}\right|^{2}\right\rfloor$.

When the starting point of the orbit is the center of the current, $R=R_{0}$ and $Z=0$ and we write the initial values of velocity components as $v_{/ /}=v_{/ / 0}$ and $v_{\perp}=v_{\perp 0}$,

$\mu B_{\phi}=\frac{m v_{\perp 0}^{2}}{2 B_{0}} \frac{B_{0} R_{0}}{R}=\frac{m v_{\perp 0}^{2}}{2}(1+x)^{-1}$,

and

$\frac{P_{\phi}-q\left(\Psi_{e x t}+\Psi_{\text {int }}\right)}{m \gamma R}=\frac{m \mathcal{v}_{/ / 0}-q R_{0} B_{V 0}\left(\psi_{\text {ext }}+\psi_{\text {int }}\right)}{m \gamma(1+x)}$.

By substituting equations (10) and (11) with (7) and (8) into equation (9), it is found that $\mathrm{W}$ has the following functional form,

$W=W\left(R_{0} B_{V 0}, \frac{R_{0}}{a}, \frac{B_{a}}{B_{V 0}}, n, v_{/ / 0}, v_{\perp 0} ; x, z\right)$.

We now can calculate the trajectory of electron projected on the poloidal cross section that starts from $\mathrm{x}=0$ and $\mathrm{z}=0$. The coordinates $\mathrm{x}$ and $\mathrm{y}$ are functions of the distance $\ell$ along the trajectory from the starting point. Then the following deferential equations describe the evolutions of $\mathrm{x}$ and $\mathrm{y}$, that is, electron trajectory in terms of normalized coordinates $\mathrm{x}$ and $\mathrm{z}$.

$\frac{d x}{d \ell}=\frac{\frac{\partial W}{\partial z}}{\sqrt{\left(\frac{\partial W}{\partial x}\right)^{2}+\left(\frac{\partial W}{\partial z}\right)^{2}}}$ and $\frac{d z}{d \ell}=-\frac{\frac{\partial W}{\partial x}}{\sqrt{\left(\frac{\partial W}{\partial x}\right)^{2}+\left(\frac{\partial W}{\partial z}\right)^{2}}}$

The functional form of $\mathrm{W}$ shown in equation (12) indicates that the normalized trajectories that start from the center of the current profile are completely determined by the set of parameters; $R_{0} B_{V 0}, R_{0} / a, B_{a} / B_{V 0}, n, v_{/ / 0}$ and $v_{\perp 0} . \quad$ It is straightforward to show that even if the starting point is different from the center the same statement holds as well for the same set of parameters.

Figure 2 shows an example of orbits of fast electrons that start with various pitch angles. In this case of $B_{a} / B_{V 0}=0.8$, there appears a small closed flux surface that looks as a tear drop in the inboard side. The field line that passes through the starting point at the center of the plasma cross section is, however, still open to the vessel wall. Nevertheless, the fast electrons that start with 
small pitch angles (5 and 6 in the figure) make confined orbits of passing particles. Note that these electrons drift forward in the sense that the direction of their currents is the same as the background current that produces $\mathrm{B}_{\mathrm{a}}$. Electrons 1 and 2 drift backward along the filed line and are immediately lost to the vessel wall. Indeed forward electrons with low velocities are lost to the vessel wall even if their pitch angles are quite small.

\section{(Figure 2)}

Figure 3 shows mappings of classification of electron orbits into passing, trapped and lost areas on the space of initial velocities of $v_{/ / 0}$ and $v_{\perp 0}$ for various parameters of $R_{0} B_{V 0}$ and $B_{a} / B_{V 0}$. Aspect ratio of current cross section is taken to be $R_{0} / a=3$ and decay index is $n=0.1$. It is noted that the characteristics depend on the product of the major radius and the magnitude of the external vertical field, $R_{0} B_{V 0}$. For example, when we assume $\mathrm{B}_{\mathrm{V} 0}=50$ Gauss, the left, center and right columns correspond the cases of $\mathrm{R}_{0}=0.3 \mathrm{~m}, 0.9 \mathrm{~m}$ and $2.7 \mathrm{~m}$, respectively. When we assume $\mathrm{R}_{0}=3 \mathrm{~m}$, the left, center and right columns correspond the cases of $\mathrm{B}_{\mathrm{V} 0}=5$ Gauss, 15 Gauss and 45 Gauss, respectively. When we assume $\mathrm{R}_{0}=1 \mathrm{~m}$, the left, center and right columns correspond the cases of $\mathrm{B}_{\mathrm{V} 0}=15$ Gauss, 45 Gauss and 135 Gauss, respectively.

\section{(Figure 3)}

Let us begin with the case shown in the left column in figure 3. Since the decay index of $B_{V}$ field is small but not zero, there is a weak mirror effect. Therefore, some electrons located near the $\mathrm{V}_{\mathrm{Z}}=0$ ellipse in the velocity space are also confined. The passing area expands toward lower energy region when the plasma current Ip starts to flow and increases as shown in the second mapping from top. In this initial stage, Ip is the equilibrium current that is proportional to the electron pressure as shown by equation (2). It is noted that the passing area at this stage is in high energy range and electrons in this area may be few since it is difficult to fill the passing area with electrons by pumping up them by ECH through a narrow path of passing area from bulk electrons. When Ip increases further by the increase of electron pressure up to a certain level (third mapping), the passing area expands up to the tail region of the bulk Maxwellian distribution. Here the current generation due to the asymmetric confinement would begin in full scale since the electron population becomes significant and the pitch angle scattering from perpendicularly EC heated electrons becomes frequent. The resultant current increase enlarges the passing area further and 
current increase would be quickly accelerated since both the electron population and pitch angle diffusion rate increase drastically towards the thermal bulk, resulting in the current jump. ECCD may also start to work at this stage and finally a closed flux surface is formed (bottom mapping). When we move to the center column, we notice that even if the self poloidal field increases to amount $80 \%$ of the $\mathrm{B}_{\mathrm{V}}$ field, the passing area does not expand to the vicinity of the bulk thermal tail. This means that when the product $R_{0} B_{V 0}$ increases current jump becomes difficult to take place. It was reported that more ECH power was needed when $\mathrm{B}_{\mathrm{V} 0}$ was increased ${ }^{[2]}$ for current jump. This result implies that in the large devices $\mathrm{B}_{\mathrm{V}}$ should be lowered. Furthermore, quickening or enhancement of pitch angle scattering by some artificial means for higher energy range of fast electrons would be beneficial to trigger positive feedback of current increase by asymmetric confinement as shown in the third mapping in this column. A superposition of a weak radial magnetic field $B_{\mathrm{r}}$ might be effective for such purpose.

Finally, importance of the equilibrium current that is driven by the bulk electrons in the open field structure should be emphasized. Confinement asymmetry of fast electrons along the field lines arises only when the equilibrium current becomes large enough to significantly modify the vacuum vertical field. The equilibrium current is proportional to the bulk electron pressure $\mathrm{P}_{\mathrm{e}}=\mathrm{n}_{\mathrm{e}} \mathrm{T}_{\mathrm{e}}$ as given by equation (2). The bulk electron density $\mathrm{n}_{\mathrm{e}}$ and temperature $\mathrm{T}_{\mathrm{e}}$ depend on many factors including the ECH power and frequency, the device scale, and the strength and the pitch (the ratio of the vertical filed strength to the toroidal field) of open helical field lines. Higher temperature generates larger current. Therefore we need better confinement even in the open filed lines and the confinement is closely related with $\mathrm{R}_{0}$ and $\mathrm{B}_{\mathrm{V} 0}$ independently ${ }^{[10]}$. Bulk electron confinement does not scale with their product. Thus, $\mathrm{R}_{0}$ and $\mathrm{B}_{\mathrm{V} 0}$ play multirole upon closing the flux surface. The effects of these factors combined with the present results for electron orbits on the formation of initial closed flux surface are issues of future work.

\section{Conclusion}

Electron orbits under the external vertical field $\left(\mathrm{B}_{\mathrm{V}}\right)$ and the self poloidal field from the toroidal plasma current are investigated by using analytic model fields for them in order to search for appearance of appropriate confinement asymmetry of electrons along the field line to ensure 
closing of field lines in ECH-started plasmas in large devices. The orbit characteristics depend on the product of the major radius and the external vertical field. This result implies that in large devices $\mathrm{B}_{\mathrm{V}}$ should be lowered. Furthermore, quickening of pitch angle scattering for higher energy range of electrons would be beneficial to trigger positive feedback of current increase by asymmetric confinement.

\section{Acknowledgments}

This work was also partially supported by the JSPS-CAS Core-University program in the field of 'Plasma and Nuclear Fusion'.

\section{REFERENCES}

[1] Nishio S, Tobita K, Konishi S, et al. 2004, Tight Aspect Ratio Tokamak Power Reactor with Superconducting TF Coils, 20th IAEA Fusion Energy Conference, Vilamoura, IAEA-CN-116/FT/P7-3, IAEA, Vienna.

[2] Yoshinaga T, Uchida M, Tanaka H, Maekawa T. 2006, Phys. Rev. Lett., 96 : 125005.

[3] Yoshinaga T, Hanada K, Sato K, et al. 2009, J. Plasma Fusion Res. SERIES 8 : 100.

[4] Tan Y, Gao Z, Wang L et al. 2010, Transient Process of a Spherical Tokamak Plasma Startup by Electron Cyclotron Waves, $23^{\text {rd }}$ IAEA Fusion Energy Conference, Daejeon, 2010 Paper EXW/P2-10.

[5] Maekawa T, Tanaka H, Uchida M. 2009, Plasma Science and Technology 11: 394.

[6] Uchida M, Yoshinaga T, Tanaka H, Maekawa T. 2010, Phys. Rev. Lett. 104: 065001.

[7] Zakharov L E, Pereverzev G V. 1988, Sov. J. Plasma Phys. 14: 75.

[8] Nishi S, Sakabe T, Uchida M, Tanaka H, Maekawa T. 2010, Plasma Phys. Control. Fusion $52: 125004$.

[9] Weston M Stacey, Jr. "FUSION PLASMA ANALYSIS” A Wiley-Interscience Publicasion John Wiley \& Sons, Inc., New York, 1981. (see p.46-p48).

[10] Parail V V, Pereverzev G V and Vojtsekhovich I A, 1985, Ionization and current drive dynamics with ECR and lower hybrid waves in a tokamak, Proc. 10th Int. Conf. on Plasma Physics and Controlled Nuclear Fusion (London, 1984) vol 1 IAEA-CN-44/F-IV-4 (Vienna: IAEA) p 605. 
Figure 1 : Coordinates

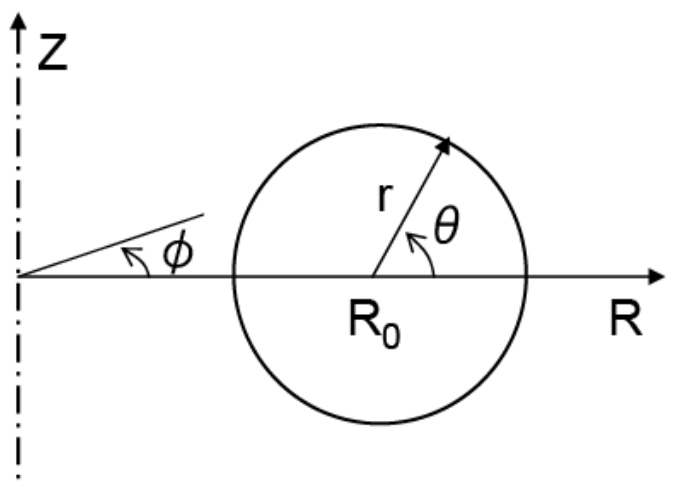

Figure 2: (a) Electron orbits projected on the poloidal cross section, and (b) initial locations of electrons in the velocity space. The electrons have the same velocity of $\mathrm{v} / \mathrm{c}=0.044$, but various pitch angles. Number 1 and 2 make lost orbits, 3and 4 do trapped orbits, and 5 and 6 do passing orbits. Aspect ratio of current cross section is $R_{0} / a=3$, decay index is $\mathrm{n}=0.1$ and $\mathrm{R}_{0} \mathrm{~B}_{\mathrm{V} 0}=45 \mathrm{mG}$, $\mathrm{B}_{\mathrm{a}} / \mathrm{B}_{\mathrm{v} 0}=0.8 \mathrm{v} / \mathrm{c}=0.044$

(a)

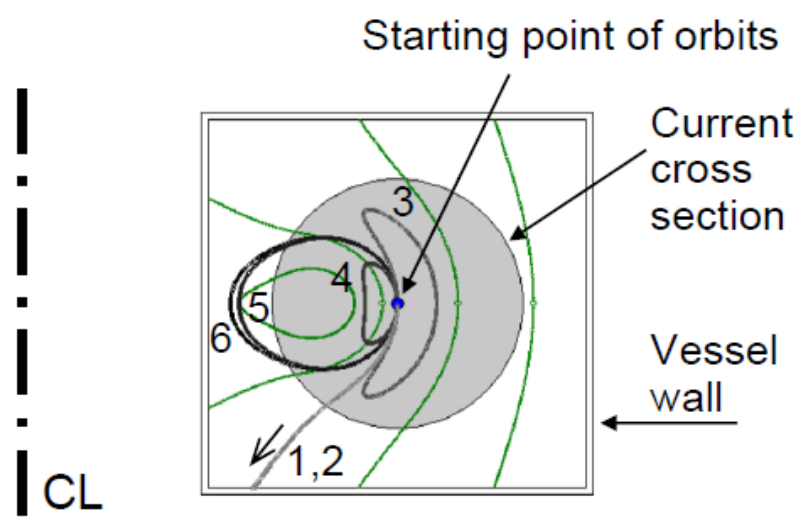

(b)




Figure 3: Mapping of electron orbits as passing, trapped and lost area on the space of initial velocities of $v_{/ / 0}$ and $v_{\perp 0}$ for various parameters of $R_{0} B_{V 0}$ and $B_{a} / B_{V 0}$. Here, $B_{a}=I_{P} / 2 \pi a$. Aspect ratio of current cross section is $R_{0} / a=3$ and decay index is $\mathrm{n}=0.1$.

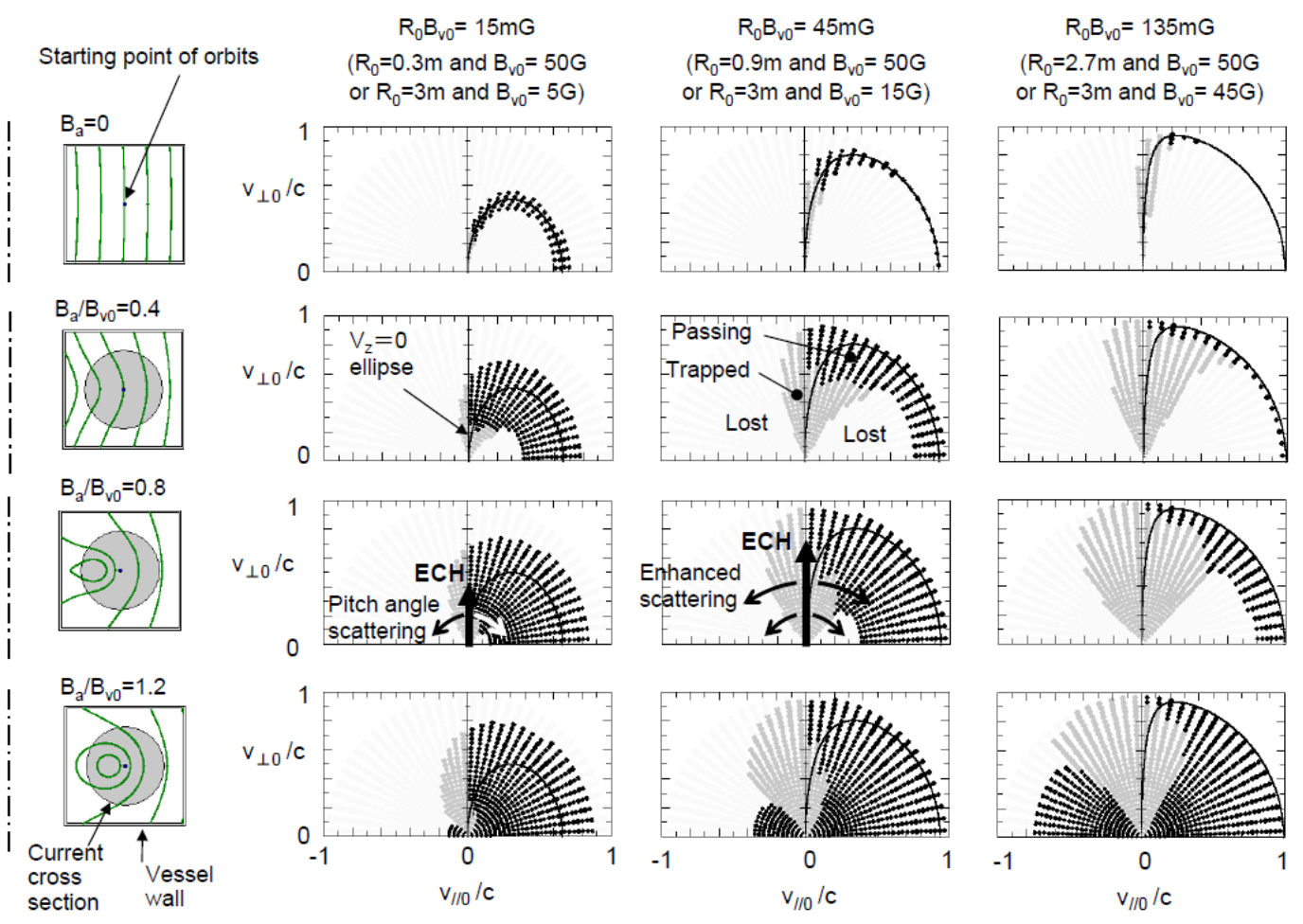

